\title{
Technical note: Cortical Thickness and Density Estimation from Clinical CT using a Prior Thickness-Density Relationship
}

\author{
Ludovic Humbert ${ }^{1}$, Javad Hazrati Marangalou², Luis Miguel del Río Barquero ${ }^{3}$, G. Harry van Lenthe ${ }^{4}$, Bert van Riet- \\ bergen ${ }^{2}$ \\ ${ }^{1}$ Galgo Medical, Barcelona, Spain \\ ${ }^{2}$ Orthopaedic Biomechanics, Department of Biomedical Engineering, Eindhoven University of Technology, Eindhoven, The Netherlands \\ ${ }^{3}$ CETIR Centre Medic, Barcelona, Spain \\ ${ }^{4}$ Biomechanics Section, KU Leuven - University of Leuven, Leuven, Belgium
}

\section{ABSTRACT}

Purpose: The cortical bone anatomical distribution is a critical component in determining the strength of bony structures. Computed Tomography $(\mathrm{CT})$ is one possible modality for analyzing the cortex in $3 \mathrm{D}$. In this paper a model-based approach for measuring the cortical bone thickness and density from clinical CT images is proposed.

Methods: Density variations across the cortex were modeled as a function of the cortical thickness and density, location of the cortex, density of surrounding tissues and imaging blur. High resolution micro-CT data of cadaver proximal femurs were analysed to determine a relationship between cortical thickness and density. This thickness-density relationship was used as a prior information to be incorporated in the model to obtain accurate measurements of cortical thickness and density from clinical CT volumes.

The method was validated using micro-CT scans of 23 cadaver proximal femurs. Simulated clinical CT images with different voxel size were generated from the micro-CT data. Cortical thickness and density measurements were obtained from the simulated images using the proposed method, and compared with micro-CT measurements to evaluate the effect of voxel size on the accuracy of the proposed method. Then, a similar comparison was performed for 19 of the 23 specimens using a clinical CT scanner. Finally, a case-control study including 25 patients with osteoporosis and 25 age-matched controls with normal bone density was performed to evaluate the proposed method in a clinical context.

Results: Cortical thickness (density) estimation errors were $0.07 \pm 0.19 \mathrm{~mm}\left(-18 \pm 92 \mathrm{mg} / \mathrm{cm}^{3}\right)$ using the simulated clinical CT volumes with the smallest voxel size (voxel size: $\left.0.33 \times 0.33 \times 0.5 \mathrm{~mm}^{3}\right)$, and $0.10 \pm 0.24 \mathrm{~mm}\left(-10 \pm 115 \mathrm{mg} / \mathrm{cm}^{3}\right) \mathrm{using}$ the volumes with the largest voxel size (voxel size: $1.0 \times 1.0 \times 3.0 \mathrm{~mm}^{3}$ ). A trend for the cortical thickness and density estimation errors to increase with voxel size was observed, and was more pronounced for thin cortices. Using clinical CT volumes of 19 of the 23 samples, an accuracy of $0.18 \pm 0.24 \mathrm{~mm}$ for the cortical thickness and $15 \pm 106 \mathrm{mg} / \mathrm{cm} 3$ for the density was found. The case-control study showed that osteoporotic patients had a thinner cortex and a lower cortical density, with average differences of $-0.8 \mathrm{~mm}$ and $-58.6 \mathrm{mg} / \mathrm{cm}^{3}$ at the proximal femur $(\mathrm{p}<0.001)$ in comparison with age-matched controls.

Conclusions: This method opens the way for the quantification of cortical bone thickness and density using clinical routine imaging techniques. Future work will concentrate on investigating how this approach can improve the estimation of mechanical strength of bony structures, the prevention of fracture, and the management of osteoporosis. 
KEYWORDS

Computed tomography, Cortical thickness, Bone mineral density, Osteoporosis, Hip fracture 


\section{INTRODUCTION}

For the year 2000, there were an estimated 9 million new osteoporotic fractures, of which 1.6 million were at the hip, 1.7 million were at the forearm and 1.4 million were clinical vertebral fractures. ${ }^{1}$ The cortical bone anatomical distribution is a critical component in determining the strength of bony structures. ${ }^{2,3}$ Quantitative Computed Tomography (QCT) is one possible modality for analyzing bony structures in 3D. QCT uses a standard X-rays CT scanner together with a calibration phantom to convert Hounsfield Units of the CT images to bone density values. However, measuring the cortical bone thickness and density from such 3D volumes or slices is not trivial. The cortical layer can be relatively thin in comparison with the image resolution, and previous studies have shown that straightforward measurement techniques of the cortical thickness and density such as the fullwidth half-maximum ${ }^{4}$ FWHM) or the $50 \%$ relative threshold ${ }^{5-7}$ methods become inaccurate when the cortex is thin in relation to the imaging resolution. In particular, cortical thickness and density measurements using clinical CT scanners are increasingly inaccurate below around $3 \mathrm{~mm}$. Many anatomical regions of interest for fracture risk assessment (femoral head, femoral neck, greater trochanter or vertebral body) exhibits cortical thicknesses below $3 \mathrm{~mm}$.

Model-based estimation methods for measuring the cortex are capable of superior accuracy. Pakdel el al. ${ }^{8}$ performed the fitting of a function of the cortical thickness and density, image blur and surrounding tissue densities to actual CT data. However, this inverse problem is ill-posed, and several studies demonstrated that parameters of the function should be constrained to guarantee accurate results. Steekstra et al. ${ }^{9}$ proposed to study the point spread function of the CT device to constrain the image blur. This process requires a phantom to be scanned, which would modify current clinical routine practices. Treece et al. ${ }^{10,11}$ proposed to hold the parameter determining the cortical density at a constraining value during the fitting process. Assuming the cortical density to be constant is however not realistic, as several studies observed a trend for cortical density to increase with thickness. ${ }^{11,12}$ In later work, Treece et al. ${ }^{12}$ accounted for this trend by modelling the cortical density as a piecewise function of the thickness. In any of these previous implementations, searching for the constraining density value ${ }^{10,11}$ or the thickness-density piecewise function parameters ${ }^{12}$ require a region of thick cortex (above around $3 \mathrm{~mm}$ ) to be present in the CT-scans . This is however not always the case, for example if only the upper part of the proximal femur (femoral head, neck and greater trochanter) is scanned. In addition, those algorithms require additional calculation steps to obtain an accurate estimation of the constraining density value or the thickness-density piecewise function, which complicated the overall process. Incorporating a constraining density value ${ }^{10,11}$ require to perform twice the fitting of a function at each node of the surface of the bone, while the algorithm relying on the thickness-density piecewise function ${ }^{12}$ requires five iterations. There is therefore a need for improved techniques for estimating the cortical thickness and density, in particular when only regions of thin cortex are present in the medical images.

In this paper a model-based approach for measuring the cortical bone thickness and density from clinical CT images is pro- 
posed. A trend for cortical density to increase with thickness was previously observed by Treece at el. ${ }^{11,12}$ In the current study, high resolution micro-CT data of cadaver proximal femurs are used to further investigate the relationship between cortical thickness and density. This thickness-density relationship is used as a prior information to obtain accurate measurements of cortical thickness and density from clinical CT volumes. Experiments with simulated clinical CT volumes are performed to evaluate the effect of voxel size on the accuracy of the proposed method. In these experiments, simulated clinical CT images with different voxel size were generated from the micro-CT data. Cortical thickness and density measurements are obtained from the simulated images using the proposed method and compared with the micro-CT measurements. Then, experiments using a clinical CT scanner were performed for 19 of the 23 cadaver proximal femurs, by comparing cortical thickness and density measurements performed from the clinical CT data with the micro-CT measurements. Finally, a case-control study comparing 25 patients with osteoporosis and 25 age-matched controls with normal bone density is performed to evaluate the proposed method in a clinical context.

\section{METHOD}

\section{II.A. Modelling density variations across the cortex}

CT data, obtained using either clinical CT or micro-CT scanners, can be sampled along a line crossing the cortex to measure density variations $\left(y_{m e s}\right)$. Similar to previous work, ${ }^{9,11,12}$ the variations of density across the cortex can be modelled, as:

$$
y_{\text {mod }}(x)=y 0+\frac{y 1-y 0}{2}\left(1+\operatorname{erf}\left(\frac{x-\left(x 1-\frac{t}{2}\right)}{\sigma \sqrt{2}}\right)\right)+\frac{y 2-y 1}{2}\left(1+\operatorname{erf}\left(\frac{x-\left(x 1+\frac{t}{2}\right)}{\sigma \sqrt{2}}\right)\right)
$$

where $x$ is the position across the cortex, $y 0, y 1$ and $y 2$ are density values in surrounding tissue, within the cortex, and within the trabecular bone respectively, $t$ is the cortical thickness, $x 1$ is the location of the center of the cortex. Following the approach taken by Prevahal et al., ${ }^{6}$ the point spread function is modelled as a normalized Gaussian function, where $\sigma$ is the standard deviation of the blur. The model in equation (1) assumes the density of the cortical layer $(y 1)$ to be uniform along the line crossing the cortex. The same assumption is made for the density of the surrounding tissue $(y 0)$ and for the density within the trabecular bone (y2). The parameters of equation (1) should be optimized so that the modelled density $y_{m o d}$ matches measured density data $y_{m e s}$ (Figure 1).

When the cortex is thick (i.e. about above $3 \mathrm{~mm}$ for typical clinical CT resolutions), the actual cortical density $y 1$ is apparent in the measured density data $y_{m e s}$, and the cortical density and thickness can be resolved unambiguously. For thinner cortical layers (as shown in Figure 1), the peak CT value is lower than the actual cortical density $y 1$, and it becomes difficult resolved the cortical density and thickness unambiguously. In the optimization process of equation (1), an increase of the cortical thickness 
estimate would have a similar effect on the density variations $y_{\bmod }$ than an increase of the cortical density estimate. Therefore prior information should be incorporated in equation (1) to constrain the model.
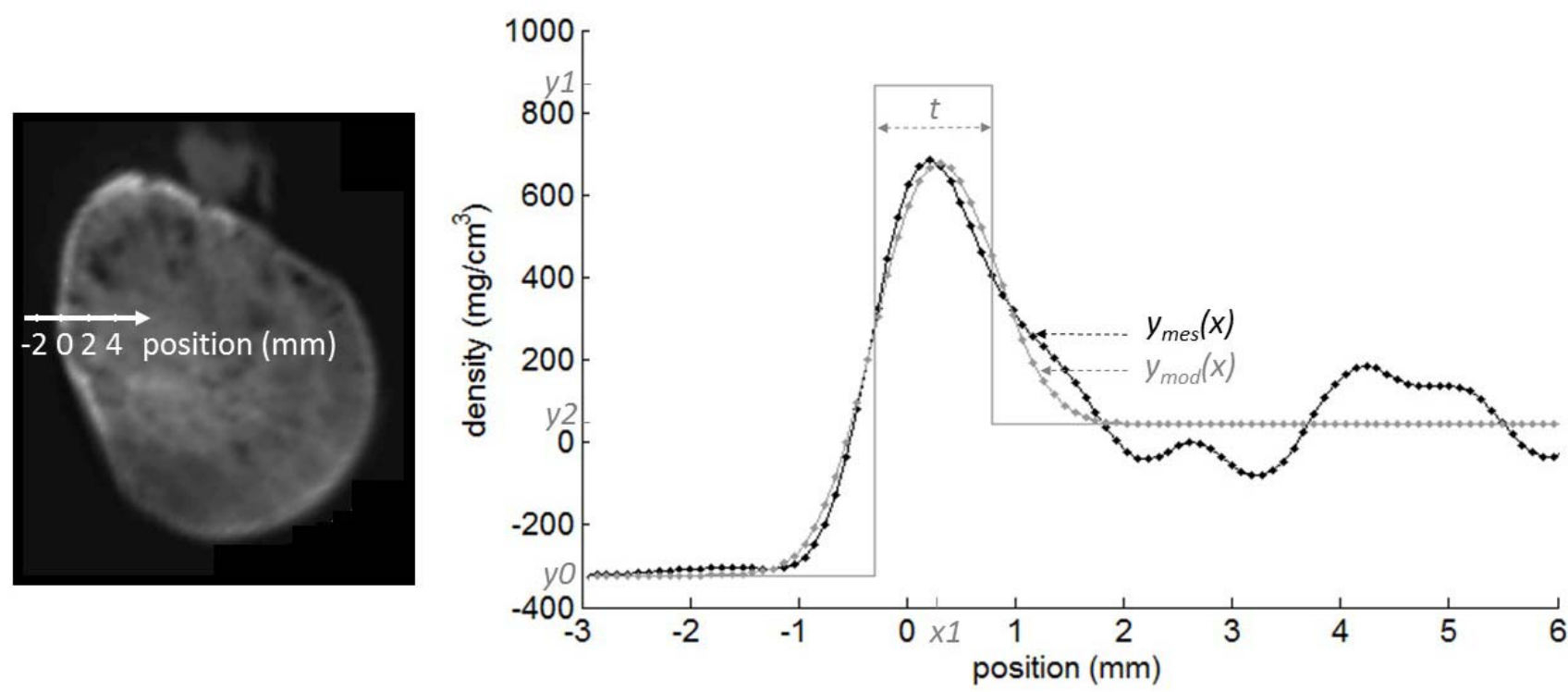

Figure 1: Measuring density variations across the cortex in a proximal femur CT slice (left). The parameters $y 0, y 1, y 2$ (density values in surrounding tissue, within the cortex, and within the trabecular bone respectively), $t$ (cortical thickness), $x 1$ (location of the center of the cortex) and $\sigma$ (imaging blur) can be optimized so that the modelled density density $y_{\text {mod }}$ matched the measured one $y_{\text {mes }}$ (right).

\section{II.B. Prior information about the thickness-density relationship}

The method presented in this paper proposes, in the case of data acquired by clinical CT, to solve equation (1) by incorporating prior information about the relationship between the cortical thickness $t$ and the cortical density $y 1$, such as:

$$
y 1=f(t)
$$

Thickness-density relationship was investigated using a database of micro-CT scans of cadaver proximal femurs collected in an earlier study. ${ }^{13}$ A database of 23 samples obtained from 8 female and 15 male donors, with a mean age of $75.0 \pm 9.1$ years [61 years - 93 years] was used. 10 were left femurs and 13 right femurs. Micro-CT scans (XtremeCT, Scanco Medical AG, Brüttisellen, Switzerland) were performed with a voxel size of $0.082 \times 0.082 \times 0.082 \mathrm{~mm}^{3}$. Images were calibrated according to the protocol recommended by the manufacturer in order to recover bone density values at each voxel. A threshold was applied to each micro-CT volume, and a 3D closing followed by a filling operation were performed to create a mask and a surface mesh over the proximal femur. At each node of the surface mesh, the normal vector to the surface was computed and 100 points were sampled along the normal. The density profile was computed by interpolating in the micro-CT volume at each sampled points. The model-based FWHM approach, as defined by Treece et al., ${ }^{12}$ was used to obtain an estimate of the cortical thickness and density each node of the surface mesh. The model proposed in Equation (1) was fitted to the measured density profile using the Levenberg-Marquardt algorithm, ${ }^{14}$ fixing $y 1$ at the maximum value observed in the density profile and optimizing all other pa- 
rameters $(y 0, y 2, t, x 1$ and $\sigma)$. The optimal value for cortical thickness $t$ and the value used for $y 1$ were stored. Cortical thick-

ness and density solutions found at each node of the surface mesh can be plotted and the average profile computed (Figure 2, left). By applying the same process to every specimens, 23 average profiles can be computed and superimposed in the same graph (Figure 2, middle). Finally, cortical thickness and density solutions computed for the 23 specimens were gathered and the average profile for the whole dataset computed (Figure 2, right). This average profile computed from the 23 specimens was stored as a look-up table, and will determine the thickness-density relationship of Equation (2).
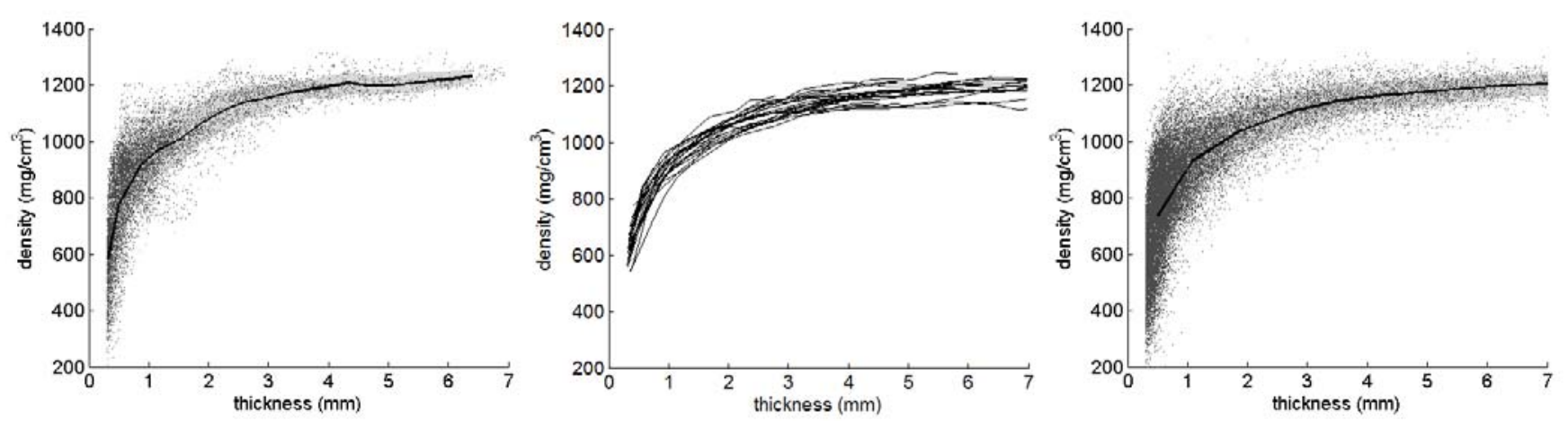

Figure 2: Cortical density against thickness computed by micro-CT, average profile and standard deviation for one specimen (left), superimposition of the density profiles computed from the 23 specimens (middle) and cortical density against thickness, average profile and standard deviation for the 23 specimens (right).

\section{II.C. Computing cortical thickness and density from clinical CT data}

Given a clinical CT volume of a bony structure, the system composed by Equations (1) and (2) is solved using the LevenbergMarquardt algorithm to fit the modelled density profile $y_{\text {mod }}$ to the measured data $y_{\text {mes }}$ (Figure 1 ). The parameters $y 0, y 1, y 2, \mathrm{t}$ and $x 1$ are optimized, while at each iteration of the optimization process, the cortical density $y 1$ is estimated using the current instance for the cortical thickness $t$ and the thickness-density look-up table.

\section{II.D. Evaluation using cadaveric specimens}

\section{II.D.1. Simulated clinical CT data}

Experiments using simulated clinical CT volumes were performed to evaluate the effect of voxel size on the accuracy of the proposed method. Low resolution CT volumes were simulated from the 23 micro-CT volumes of cadaveric proximal femur specimens. A bicubic interpolation was performed in the original micro-CT volumes (voxel size: $0.082 \times 0.082 \times 0.082 \mathrm{~mm}^{3}$ ) to generate volumes with the following characteristics: voxel size in the transverse plane of $0.33 \mathrm{~mm} \times 0.33 \mathrm{~mm}$, $0.66 \mathrm{~mm} \times 0.66 \mathrm{~mm}$ and $1.00 \mathrm{~mm} \times 1.00 \mathrm{~mm}$ and voxel size along the superior-inferior axis of $0.5 \mathrm{~mm}, 1.0 \mathrm{~mm}, 2.0 \mathrm{~mm}$ and $3.0 \mathrm{~mm}$. The accuracy of the cortical thickness and density calculation method was evaluated using a leave-one-out cross valida- 
the micro-CT high resolution volume of the $i^{\text {th }}$ specimen was used to measure the cortical thickness and density at each node of the femoral shape. Using the remaining 22 micro-CT samples, a density-thickness relationship was determined following the pipeline described in section 0 . This density-thickness relationship was used as a prior information for the current method (section II.C) to estimate the cortical thickness and density from the simulated clinical CT volumes generated for the $i^{\text {th }}$ specimen. Cortical thickness and density estimated from the simulated clinical CT volumes were compared with those computed using the high resolution micro-CT volume, and the process was repeated for the 23 samples in order to evaluate the accuracy of the method over the whole dataset.

\section{II.D.2. Clinical CT data}

19 of the 23 cadaveric proximal femur specimens were scanned using a clinical CT scanner. The 19 samples were obtained from 5 female and 14 male donors, with a mean age of $74.1 \pm 9.2$ years [61 years -93 years]. 10 were left femurs and 9 right femurs. A Siemens SOMATOM Definition Flash CT scanner (Siemens Healthcare, Erlangen, Germany) was used. The scans were acquired with a pixel size of $0.30 \mathrm{~mm} \times 0.30 \mathrm{~mm}$, a slice thickness of $0.60 \mathrm{~mm}$ and a distance between consecutive slices of $0.30 \mathrm{~mm}$. A QRM-Forearm-Phantom (QRM GmbH., Möhrendorf, Germany) calibration phantom was scanned together with the cadaveric specimens, and used to convert the Hounsfield units at each pixel into bone density values according to the protocol recommended by the manufacturer. A surface mesh was obtained for each proximal femur sample by using the segmentation protocol described in section II.B, and the cortical thickness and density was estimated using the proposed method. The leaveone-out cross validation approach described in section II.D was used, meaning that the cortical thickness and density estimations performed for the $i^{\text {th }}$ specimen relied on a density-thickness relationship built over a database excluding the $i^{\text {th }}$ specimen. To compare the clinical CT with micro-CT measurements, each clinical CT surface mesh was rigidly registered onto the corresponding micro-CT surface mesh, using the iterative closest point method. ${ }^{15}$ Cortical thickness and density estimation performed at each node of the registered clinical CT surface mesh was compared with the micro-CT measurements at the closest node of the micro-CT surface mesh.

\section{II.E. Clinical experiments}

A case-control clinical study was performed to evaluate the performance of the proposed method in a clinical context. 25 patients with osteoporosis together with 25 age-matched controls with normal bone density were collected by CETIR Grup Mèdic (Barcelona, Spain). Written informed consent was obtained from all patients. Dual-energy X-ray Absorptiometry scans were performed to compute areal Bone Mineral Density values and T-scores at the proximal femur, and identify patients with osteoporosis and normal bone density, following the World Health Organization classification method ${ }^{16}$ and International Society For Clinical Densitometry recommendations. ${ }^{17}$ For each osteoporotic patient, a subject with normal bone density, same gender and same age \pm 5 years was chosen. QCT-scans were performed using a Philips Gemini GXL 16 (Philips Healthcare, Best, The 
Netherlands), a Discovery CT750 HD scanner or a HiSpeed QX/I scanner (GE Healthcare, Madison, WI). The region of interest of the scans included the proximal extremity of the femur (femoral head, neck and greater trochanter). Voxel size ranged from $0.68 \times 0.68 \times 0.5 \mathrm{~mm}^{3}$ to $1.06 \times 1.06 \times 0.5 \mathrm{~mm}^{3}$. A calibration phantom (Mindways Software Inc., Austin, TX) was used and the Hounsfield units at each voxel were converted to bone density values according to the protocol recommended by the manufacturer. A surface mesh over the left proximal femur was generated from the QCT acquisitions using a similar approach to the one used to segment the micro-CT data (section II.B). The cortical thickness and density distribution was computed using the proposed method (section II.C), including the prior thickness-density relationship determined from the micro-CT volumes of the 23 cadaveric proximal femur specimens (section II.B), and results obtained for both groups (osteoporotic patients and control group) were compared. For comparison purposes, a reference surface mesh was chosen among the database, and a non-rigid point set registration algorithm ${ }^{18}$ was used to register each surface mesh of the database onto the reference mesh. The registered surface meshes were used to establish a point correspondence between the reference surface mesh and the surface meshes of the database, and display the average cortical thickness and density distribution for both groups over the reference surface mesh.

\section{RESULTS}

\section{III.A. Evaluation using cadaveric specimens}

A comparison between the cortical thickness and density computed using micro-CT (FWHM approach), simulated clinical CT data, and clinical CT data is proposed for one sample in Figure 3.
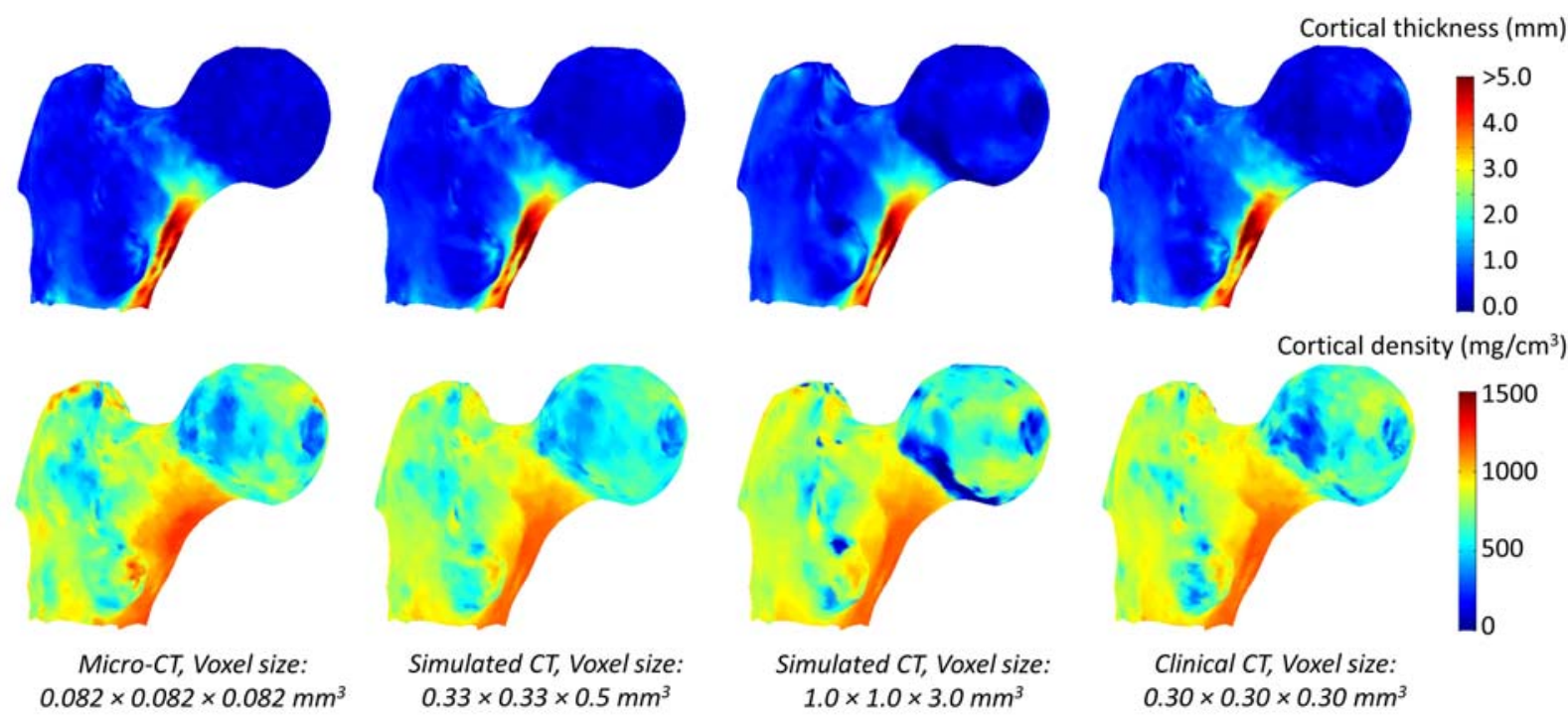

Simulated CT, Voxel size $0.33 \times 0.33 \times 0.5 \mathrm{~mm}^{3}$
$0.30 \times 0.30 \times 0.30 \mathrm{~mm}^{3}$

Figure 3: Color maps over the femoral shape showing the cortical thickness (top) and density (bottom) computed using micro-CT, simulated CT and clinical CT data for one sample 
The mean ( \pm standard deviation) accuracy computed over the 23 samples was $0.07 \pm 0.19 \mathrm{~mm}$ for the cortical thickness and 175 $18 \pm 92 \mathrm{mg} / \mathrm{cm}^{3}$ for the density, when computed for cortices in the range $[0.3 \mathrm{~mm}, 6.0 \mathrm{~mm}]$ and using the simulated clinical CT volumes with the smallest voxel size $\left(0.33 \times 0.33 \times 0.5 \mathrm{~mm}^{3}\right)$. Using the volumes with the largest voxel size $\left(1.0 \times 1.0 \times 3.0 \mathrm{~mm}^{3}\right)$, the accuracy was $0.10 \pm 0.24 \mathrm{~mm}$ and $-10 \pm 115 \mathrm{mg} / \mathrm{cm}^{3}$. Using the real clinical CT volumes of $19 \mathrm{of}$ the 23 samples, an accuracy of $0.18 \pm 0.24 \mathrm{~mm}$ for the cortical thickness and $15 \pm 106 \mathrm{mg} / \mathrm{cm} 3$ for the density was found, when computed for cortices in the range $[0.3 \mathrm{~mm}, 6.0 \mathrm{~mm}]$. Detailed results for cortices in the range $[0.3 \mathrm{~mm}, 1.0 \mathrm{~mm}[,[1.0 \mathrm{~mm}, 3.0$ $\mathrm{mm}[$ and $[3.0 \mathrm{~mm}, 6.0 \mathrm{~mm}]$ are provided in Table 1.

Table 1: Cortical thickness and density estimation accuracy (Mean \pm Standard deviation) computed using the simulated clinical CT and real clinical CT volumes, and comparison with results provided by Treece et al. ${ }^{12}$

\begin{tabular}{|c|c|c|c|c|c|c|c|c|}
\hline \multirow[b]{3}{*}{ Voxel size $\left(\mathrm{mm}^{3}\right)$} & \multicolumn{4}{|c|}{ Cortical thickness (mm) } & \multicolumn{4}{|c|}{ Density $\left(\mathrm{mg} / \mathrm{cm}^{3}\right)$} \\
\hline & \multicolumn{4}{|c|}{ Thickness range (mm) } & \multicolumn{4}{|c|}{ Thickness range (mm) } \\
\hline & {$[3.0,6.0]$} & {$[1.0,3.0[$} & {$[0.3,1.0[$} & {$[0.3,6.0]$} & {$[3.0,6.0]$} & {$[1.0,3.0[$} & {$[0.3,1.0[$} & {$[0.3,6.0]$} \\
\hline \multicolumn{9}{|c|}{ Simulated Clinical CT volumes $(\mathrm{N}=23)$} \\
\hline $0.33 \times 0.33 \times 0.5=0.05$ & $-0.03 \pm 0.31$ & $0.04 \pm 0.23$ & $0.10 \pm 0.13$ & $0.07 \pm 0.19$ & $-3 \pm 47$ & $-6 \pm 77$ & $-25 \pm 101$ & $-18 \pm 92$ \\
\hline $0.33 \times 0.33 \times 1.0=0.11$ & $-0.04 \pm 0.32$ & $0.04 \pm 0.23$ & $0.11 \pm 0.14$ & $0.08 \pm 0.20$ & $-3 \pm 47$ & $-5 \pm 76$ & $-20 \pm 103$ & $-15 \pm 93$ \\
\hline $0.33 \times 0.33 \times 2.0=0.22$ & $-0.05 \pm 0.32$ & $0.05 \pm 0.24$ & $0.11 \pm 0.16$ & $0.08 \pm 0.21$ & $-4 \pm 48$ & $-4 \pm 77$ & $-20 \pm 111$ & $-14 \pm 99$ \\
\hline $0.33 \times 0.33 \times 3.0=0.33$ & $-0.06 \pm 0.33$ & $0.05 \pm 0.26$ & $0.11 \pm 0.17$ & $0.08 \pm 0.22$ & $-4 \pm 47$ & $-4 \pm 78$ & $-29 \pm 127$ & $-20 \pm 111$ \\
\hline $0.66 \times 0.66 \times 0.5=0.22$ & $-0.06 \pm 0.33$ & $0.05 \pm 0.26$ & $0.11 \pm 0.17$ & $0.08 \pm 0.22$ & $-4 \pm 47$ & $-4 \pm 77$ & $-25 \pm 124$ & $-18 \pm 108$ \\
\hline $0.66 \times 0.66 \times 1.0=0.44$ & $-0.06 \pm 0.33$ & $0.05 \pm 0.26$ & $0.12 \pm 0.17$ & $0.08 \pm 0.22$ & $-4 \pm 47$ & $-4 \pm 77$ & $-21 \pm 121$ & $-15 \pm 107$ \\
\hline $0.66 \times 0.66 \times 2.0=0.87$ & $-0.06 \pm 0.33$ & $0.06 \pm 0.26$ & $0.12 \pm 0.18$ & $0.09 \pm 0.23$ & $-4 \pm 47$ & $-3 \pm 77$ & $-19 \pm 122$ & $-13 \pm 107$ \\
\hline $0.66 \times 0.66 \times 3.0=1.31$ & $-0.07 \pm 0.34$ & $0.06 \pm 0.26$ & $0.12 \pm 0.18$ & $0.09 \pm 0.23$ & $-4 \pm 47$ & $-3 \pm 78$ & $-21 \pm 130$ & $-15 \pm 113$ \\
\hline $1.0 \times 1.0 \times 0.5=0.50$ & $-0.07 \pm 0.34$ & $0.06 \pm 0.27$ & $0.13 \pm 0.18$ & $0.09 \pm 0.23$ & $-4 \pm 47$ & $-2 \pm 77$ & $-18 \pm 128$ & $-13 \pm 112$ \\
\hline $1.0 \times 1.0 \times 1.0=1.00$ & $-0.07 \pm 0.34$ & $0.06 \pm 0.27$ & $0.13 \pm 0.19$ & $0.09 \pm 0.24$ & $-4 \pm 47$ & $-2 \pm 77$ & $-15 \pm 127$ & $-11 \pm 111$ \\
\hline $1.0 \times 1.0 \times 2.0=2.00$ & $-0.07 \pm 0.34$ & $0.07 \pm 0.27$ & $0.14 \pm 0.19$ & $0.10 \pm 0.24$ & $-4 \pm 47$ & $-1 \pm 77$ & $-13 \pm 128$ & $-9 \pm 111$ \\
\hline $1.0 \times 1.0 \times 3.0=3.00$ & $-0.07 \pm 0.34$ & $0.07 \pm 0.27$ & $0.14 \pm 0.20$ & $0.10 \pm 0.24$ & $-4 \pm 47$ & $-1 \pm 78$ & $-14 \pm 133$ & $-10 \pm 115$ \\
\hline \multicolumn{9}{|l|}{ Clinical CT volumes $(\mathrm{N}=19)$} \\
\hline $0.30 \times 0.30 \times 0.30=0.03$ & $0.09 \pm 0.42$ & $0.16 \pm 0.28$ & $0.20 \pm 0.17$ & $0.18 \pm 0.24$ & $5 \pm 49$ & $11 \pm 84$ & $18 \pm 119$ & $15 \pm 106$ \\
\hline \multicolumn{9}{|c|}{ Treece et al. ${ }^{12}$ 'CBM v3' method } \\
\hline $0.33 \times 0.33 \times 1.0=0.33$ & $0.04 \pm 0.25$ & $0.26 \pm 0.40$ & $0.04 \pm 0.31$ & & $-27 \pm 109$ & $-89 \pm 160$ & $-30 \pm 264$ & \\
\hline
\end{tabular}

$0.33 \times 0.33 \times 1.0 \mathrm{~mm}^{3}, 0.66 \times 0.66 \times 2.0 \mathrm{~mm}^{3}$ and $1.0 \times 1.0 \times 3.0 \mathrm{~mm}^{3}$ ) and from the clinical CT dataset is plotted against the thickness calculated from the micro-CT volumes ('true thickness'). The mean values together with the $40 \%, 68 \%$ (equivalent to a one-standard-deviation range), $80 \%$ and $95 \%$ confidence intervals are shown. A similar plot is provided for the cortical density (Figure 5). In Figure 6 are plotted the cortical density errors (estimated density - true density) against the true thickness. 

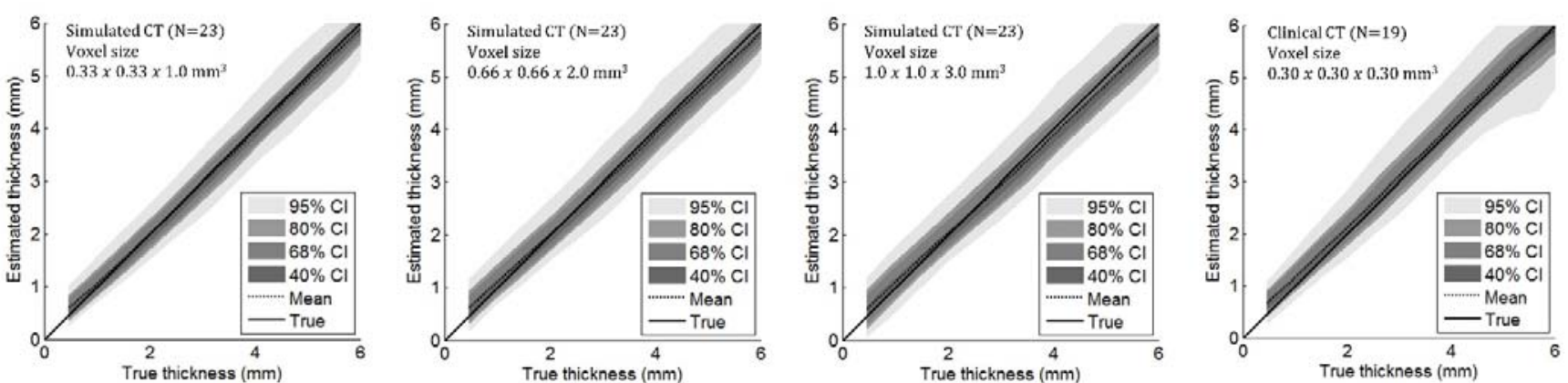

Figure 5: Density estimated from three of the clinical CT dataset (mean and confidence intervals) against true density
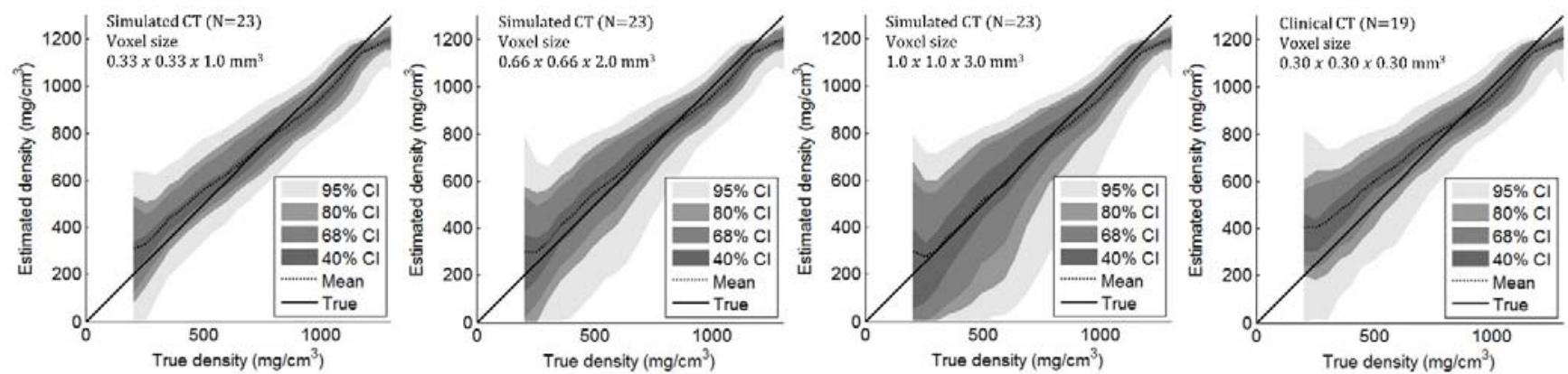

Figure 6: Differences between density estimated from three of the clinical CT dataset and true density (mean and confidence intervals) against true thickness
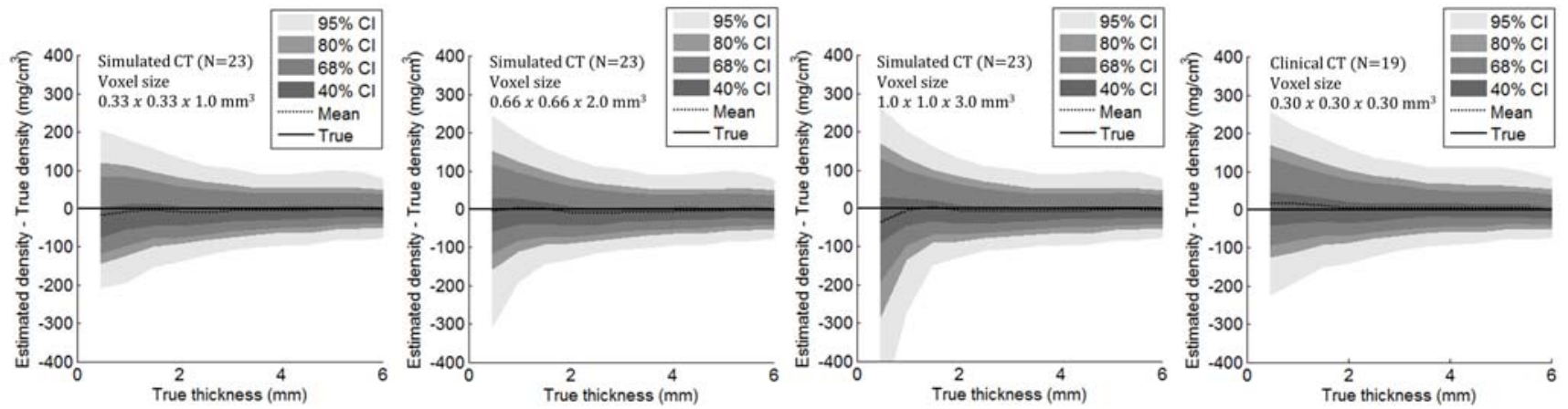

\section{III.B. Clinical experiments}

No statistical difference was found in terms of age, weight, height and body mass index between patients with osteoporosis and control group (p-values $>0.05$, Student's t-test) (Table 2). However, the average cortical thickness was found to be significantly lower for osteoporotic patients $(0.84 \pm 0.59 \mathrm{~mm})$ in comparison with control group $(1.02 \pm 0.73 \mathrm{~mm})(\mathrm{p}$-value $=0.000)$ at the total femur region. Similar results were found for the density of the cortex $\left(752.3 \pm 220.4 \mathrm{mg} / \mathrm{cm}^{3}\right.$ for patients with osteoporosis against $810.9 \pm 200.6 \mathrm{mg} / \mathrm{cm}^{3}$ for control group, $\mathrm{p}$-value $\left.=0.000\right)$. Differences for the cortical thickness were found to be greater at the neck $(-0.29 \mathrm{~mm})$ and trochanter $(-0.25 \mathrm{~mm})$ than at the head $(-0.08 \mathrm{~mm})$. In particular, Figure 7 shows that the greatest differences for the cortical thickness were found at the medial part of the neck and trochanter. However, when computed as percentages, the differences were found to be rather homogenously distributed over the femoral shape (Figure 7), with average differences between $-11.2 \%$ and $-17.7 \%$ depending on the region of interest (Table 2). Differences for the cortical density were 
210 found to be slightly higher at the femoral head $\left(-64.0 \mathrm{mg} / \mathrm{cm}^{3},-8.7 \%\right)$ than at the neck $\left(-61.9 \mathrm{mg} / \mathrm{cm}^{3},-6.5 \%\right)$ and trochanter ($\left.50.4 \mathrm{mg} / \mathrm{cm}^{3},-5.3 \%\right)$. 
Table 2: Population characteristics, cortical thickness and density for osteoporosis and control groups (Mean \pm Standard Deviation (SD) values and p-value from Student's t-test)

\begin{tabular}{|c|c|c|c|c|}
\hline & $\begin{array}{l}\text { Control group }(\mathrm{N}=25) \\
\text { Mean } \pm \mathrm{SD}\end{array}$ & $\begin{array}{l}\text { Osteoporosis group }(\mathrm{N}=25) \\
\text { Mean } \pm \text { SD }\end{array}$ & p-value & Differences \\
\hline Age (years) & $54.1 \pm 10.2$ & $54.5 \pm 10.6$ & 0.893 & - \\
\hline Weight (kg) & $64.1 \pm 10.2$ & $62.8 \pm 10.6$ & 0.651 & - \\
\hline Height (cm) & $162.1 \pm 7.2$ & $163 \pm 9.4$ & 0.697 & - \\
\hline Body mass index $\left(\mathrm{kg} / \mathrm{m}^{2}\right)$ & $24.4 \pm 3.7$ & $23.6 \pm 3.5$ & 0.441 & - \\
\hline \multicolumn{5}{|l|}{ Cortical thickness (mm) } \\
\hline Total Femur & $1.02 \pm 0.73$ & $0.84 \pm 0.59$ & 0.000 & $-0.18(-16.2 \%)$ \\
\hline Head & $0.58 \pm 0.24$ & $0.51 \pm 0.22$ & 0.000 & $-0.08(-11.2 \%)$ \\
\hline Neck & $1.49 \pm 0.96$ & $1.20 \pm 0.81$ & 0.000 & $-0.29(-17.7 \%)$ \\
\hline Trochanter & $1.34 \pm 0.71$ & $1.09 \pm 0.56$ & 0.000 & $-0.25(-17.3 \%)$ \\
\hline \multicolumn{5}{|l|}{ Density $\left(\mathrm{mg} / \mathrm{cm}^{3}\right)$} \\
\hline Total Femur & $810.9 \pm 200.6$ & $752.3 \pm 220.4$ & 0.000 & $-58.6(-7.0 \%)$ \\
\hline Head & $679.6 \pm 176.5$ & $615.5 \pm 200.7$ & 0.000 & $-64.0(-8.7 \%)$ \\
\hline Neck & $916.8 \pm 180.4$ & $854.9 \pm 207.8$ & 0.000 & $-61.9(-6.5 \%)$ \\
\hline Trochanter & $923.5 \pm 126.1$ & $873.1 \pm 136.8$ & 0.000 & $-50.4(-5.3 \%)$ \\
\hline
\end{tabular}

\section{Cortical thickness $(\mathrm{mm})$}

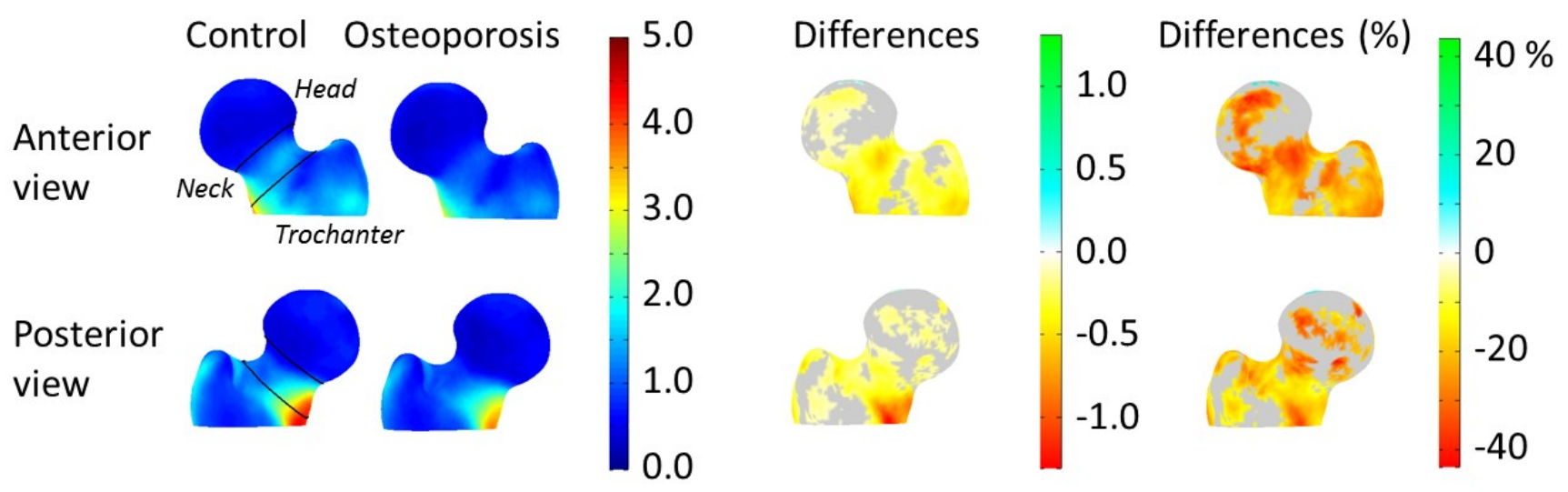

Cortical density $\left(\mathrm{mg} / \mathrm{cm}^{3}\right)$

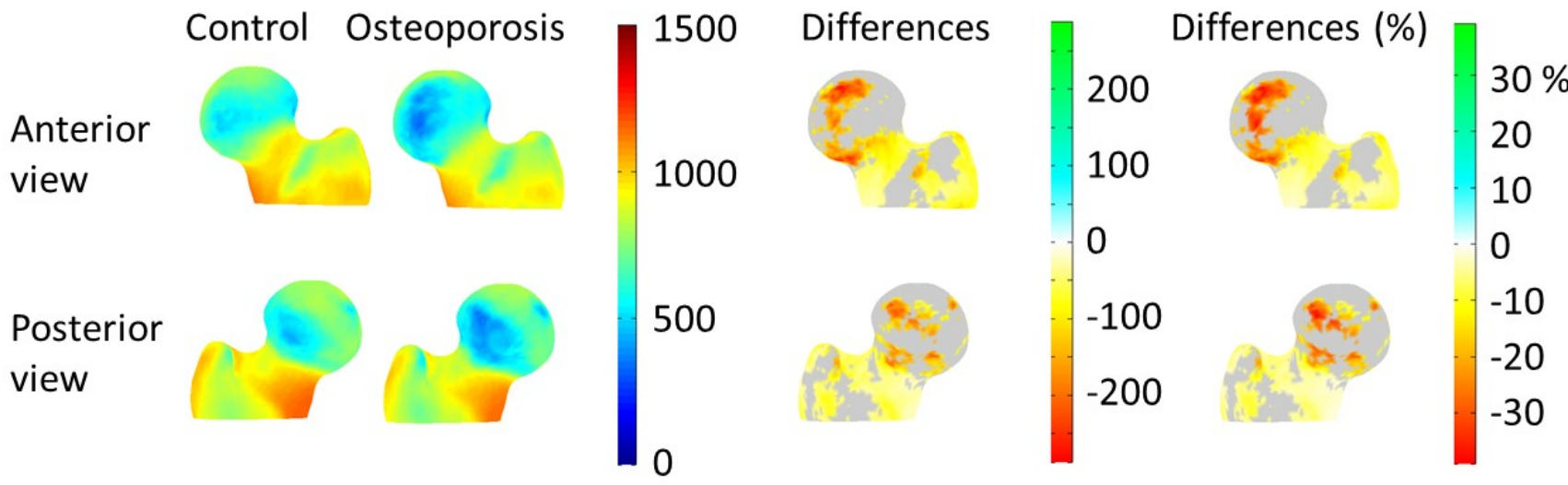

Figure 7: Average cortical thickness (top) and density (bottom) for the 25 osteoporotic patients and 25 controls. The region of interest (Head, Neck and Trochanter) are displayed (top left). Differences are showed as absolute values and percentages when statistically significant (p-values $<0.05$, Student's t-test, in grey for p-values $>0.05)$. 


\section{DISCUSSION}

The standard deviations of the estimation errors were higher when computed for thick cortex (between $0.32 \mathrm{~mm}$ and $0.35 \mathrm{~mm}$ in the range $[3.0 \mathrm{~mm}, 6.0 \mathrm{~mm}]$ ) than for thin cortex (between $0.16 \mathrm{~mm}$ and $0.21 \mathrm{~mm}$ in the range [0.3 $\mathrm{mm}, 1.0 \mathrm{~mm}]$, Table 1 ). In the case of the cortical density, the bias and estimation errors were higher when measured for low density cortices (Figure 5) or for thin cortices (Figure 6), which agree with previous work. ${ }^{12}$ Figure 4 and Figure 6 show however that the proposed method provides a relatively unbiased and rather accurate estimation for the cortical thickness and density in the whole thickness range $[0.3,6.0]$.

As would be expected, a general trend for the cortical thickness and density estimation errors to increase with voxel size was observed. Increase in voxel size had a greater impact on thin cortex. Standard deviations for cortical thickness (density) estimation errors ranged between $0.31 \mathrm{~mm}$ to $0.34 \mathrm{~mm}\left(47 \mathrm{mg} / \mathrm{cm}^{3}\right.$ to $\left.48 \mathrm{mg} / \mathrm{cm}^{3}\right)$ for cortices in the range [3.0 mm, $6.0 \mathrm{~mm}$ ], while higher variations were observed for thin cortices $([0.3 \mathrm{~mm}, 1.0 \mathrm{~mm}])$, with standard deviations between $0.13 \mathrm{~mm}$ to $0.20 \mathrm{~mm}$ $\left(101 \mathrm{mg} / \mathrm{cm}^{3}\right.$ to $133 \mathrm{mg} / \mathrm{cm}^{3}$ ) (Table 1$)$. However, the differences in terms of estimation errors between the lowest and highest resolution clinical CT volumes were found to be relatively low, considering that the voxels of the lowest resolution volumes $\left(1.0 \times 1.0 \times 3.0=3.0 \mathrm{~mm}^{3}\right)$ were 55 times bigger than the ones of the highest resolution volumes $(0.33 \times 0.33 \times 0.5=$ $0.05 \mathrm{~mm}^{3}$ ). The bias was also rather low for all the tested configurations (between $0.07 \mathrm{~mm}$ and $0.10 \mathrm{~mm}$ for the cortical thickness, and between -9 and $-20 \mathrm{mg} / \mathrm{cm}^{3}$ for the density, when observed for cortices in the range $[0.3,6.0]$ ). This shows the ability of the method to provide an accurate estimation for the femoral cortical thickness and density, even from low resolution CT data.

In previous work by Treece et al., ${ }^{12}$ several methods for cortical thickness and density estimation were evaluated and compared, including the FWHM method, ${ }^{5}$ the 'preset blur' method, which rely on a prior estimate of the blur, the 'nothing preset' approach as proposed by Pakdel et al., ${ }^{8}$ the 'CBM v1' method as proposed by Treece et al. ${ }^{11}$ and the new 'CBM v2' and 'CBM v3' methods introduced in Treece et al. ${ }^{12}$ The evaluation was performed using 70 femur specimens. Cortical thickness and density estimated from clinical CT volumes (voxel size: $0.33 \times 0.33 \times 1.0 \mathrm{~mm}^{3}$ ) were compared with calculation from micro-CT volumes (voxel size: $0.082 \times 0.082 \times 0.082 \mathrm{~mm}^{3}$ ). The 'CBM v3' method was found to provide the best results in estimating the cortical thickness and density from clinical CT data, and the main results of this evaluation are summed-up in Table 1. Although the samples included in both studies were different, this comparison shows that our approach provides an estimation of the cortical thickness with a similar accuracy in comparison with the ' $\mathrm{CBM}$ v3' method. With regards to the density estimation, the current implementation showed lower bias and errors (standard deviation) than the 'CBM v3' method.

As previously mentioned, state-of-the-art algorithms for cortical thickness and/or density estimation ${ }^{10-12}$ require the analysis of regions of thick cortex (typically cortex above $3 \mathrm{~mm}$ ) in the CT data. If no region of thick cortex is present in the image, such algorithms cannot be used in a straightforward manner to provide an estimation of the cortical thickness and density. One inter- 
esting aspect of the proposed approach is that the cortical thickness and density can be computed even if no region of thick cortex is present in the image. Figure 8 shows a comparison between cortical thickness and density computed using high resolution micro-CT and low resolution volumes for one femur sample. The analysis was limited to the upper part of the proximal femur, where the maximal value for cortical thickness was $2.97 \mathrm{~mm}$. This figure show that, even in the absence of region of thick cortex, an accurate estimation of the cortical thickness and density can be obtained. In the database of 25 osteoporotic patients included in the clinical experiments, $20 \%$ of the proximal femurs exhibited a cortex with a maximal thickness lower than $3 \mathrm{~mm}$.
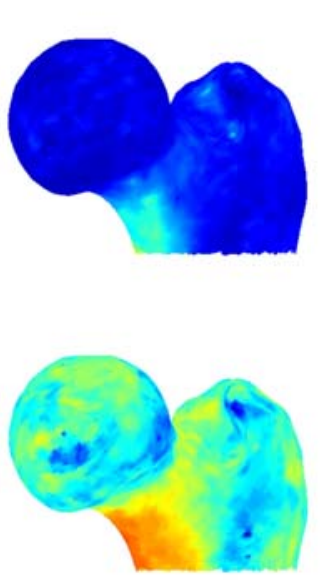

Micro-CT, Voxel size: $0.082 \times 0.082 \times 0.082 \mathrm{~mm}^{3}$
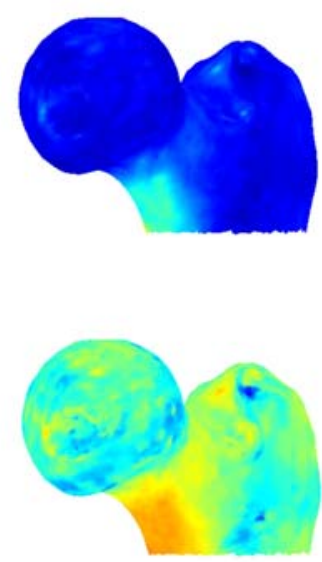

Simulated CT, Voxel size: $0.33 \times 0.33 \times 0.5 \mathrm{~mm}^{3}$
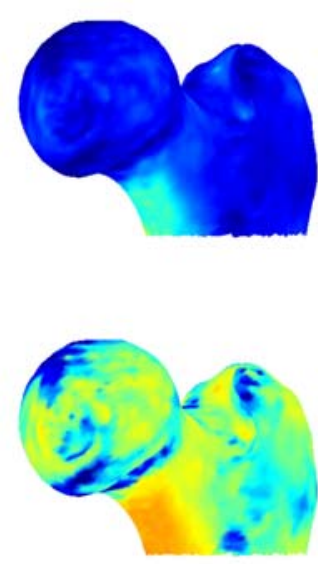

Simulated CT, Voxel size: $1.0 \times 1.0 \times 3.0 \mathrm{~mm}^{3}$
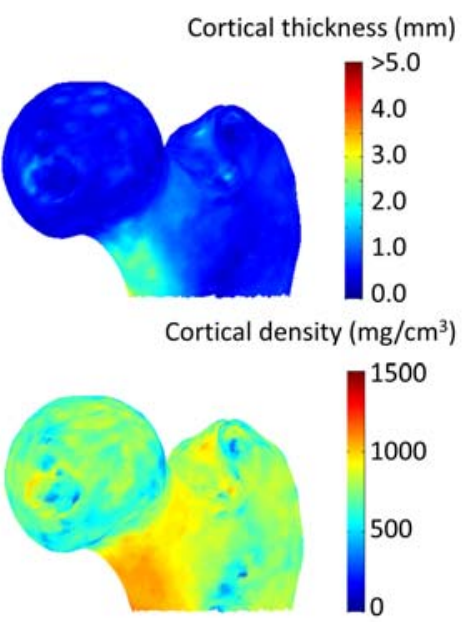

Clinical CT, Voxel size: $0.30 \times 0.30 \times 0.30 \mathrm{~mm}^{3}$

Figure 8: Color maps over the femoral shape showing the cortical thickness (top) and density (bottom) computed using micro-CT, simulated CT and clinical CT data for one sample. Maximum value for cortical thickness computed from the micro-CT volume was $2.97 \mathrm{~mm}$.

The use of simulated data allow us to evaluate a large number of configurations in terms of image resolution. The proposed cortical thickness and density calculation method was evaluated for 12 voxel sizes ranging from $0.33 \times 0.33 \times 0.5 \mathrm{~mm}^{3}$ to $1.0 \times 1.0 \times 3.0 \mathrm{~mm}^{3}$ and representing typical CT clinical routine settings for proximal femur assessment. ${ }^{19}$ An evaluation of our approach using real clinical CT data is expected to show different results in terms of accuracy. Differences between micro-CT and clinical CT calibration protocols (to recover bone density values from the raw CT data) are expected to introduce a systematic error (bias). However, the standard deviation of the thickness and density estimation errors should not be affected. On the other hand, the clinical CT images will differ from the ones that were generated in this study by sampling in the micro-CT volumes, and will depend on the physical properties and settings of the CT device. As the cortical thickness and density estimation errors largely depend on the imaging blur, which is highly correlated with the resolution of the images, similar trends should be observed when using our approach from real clinical CT data. This should however be further investigated. 
The thickness-density relationship (section II.B) was determined using cadaveric specimens in order to perform high precision micro-CT measurements. The samples were fresh femurs, stored frozen and thawed to room temperature before being scanned. Previous studies demonstrated that this procedure does not affect neither the trabecular nor the cortical bone mineralization. ${ }^{20,21}$ Therefore it is very likely that a similar thickness-density relationship would have been found if it would have been possible to perform such micro-CT scans in vivo.

The thickness-density relationship was computed by generating an average profile over the data collected for the 23 cadaveric specimens. The average profile was very similar when computed using only data from male $(\mathrm{n}=15)$ or female donors $(\mathrm{n}=8)$. No information was available about any pathology affecting the bone structure, such as osteoporosis or osteoarthritis. Therefore it was not possible to investigate to what extent the relationship between density and thickness may depend upon the patient's disease. However, the state of these diseases is correlated with the age of the patient, ${ }^{16}$ and the database of specimens included donors from 61 years to 93 years. No trend for the thickness-density relationship to depend upon the age of the patient was observed. This should be, however, further investigated using a larger database of cadaveric specimens.

Numerous case-control studies have shown that osteoporotic patients with a high risk of fracture have a thinner cortex and a lower cortical density. ${ }^{22-26}$ The clinical experiment conducted in this study showed the ability of the proposed method to highlight similar findings between patients with osteoporosis and controls collected using clinical routine QCT protocols. Yang et al. ${ }^{26}$ found differences between $-0.1 \mathrm{~mm}(-14 \%)$ and $-0.3 \mathrm{~mm}(-23 \%)$ for the cortical thickness at the femoral neck, and differences of $-97 \mathrm{mg} / \mathrm{cm}^{3}(-17 \%)$ for the cortical density between patient with osteoporotic fracture and age-matched controls. Johannesdottir et al. ${ }^{25}$ measured differences of $-0.23 \mathrm{~mm}(-8 \%)$ at the femoral neck between patients with neck fracture osteoporotic fracture at the neck and age-matched controls. In the clinical experiment conducted in this study, differences at the femoral neck were $-0.29 \mathrm{~mm}(-17.7 \%)$ for the cortical thickness and $-61.9 \mathrm{mg} / \mathrm{cm}^{3}(-6.5 \%)$ for the density. However, comparison with previous work is not trivial as the characteristics of the populations, study design and methods to analyze the cortex differ from a study to another.

To conclude, a model-based approach for measuring the cortical bone thickness and density from clinical low resolution images, incorporating a prior thickness-density relationship computed using high resolution micro-CT data, was introduced. The method was evaluated against micro-CT using simulated clinical CT data, with cortical thickness estimation errors between 0.07 $\pm 0.19 \mathrm{~mm}$ and $0.10 \pm 0.24 \mathrm{~mm}$ and cortical density estimation errors between $-18 \pm 92 \mathrm{mg} / \mathrm{cm}^{3}$ and $-10 \pm 115 \mathrm{mg} / \mathrm{cm}^{3} . \mathrm{This}$ method opens the way for the quantification of cortical bone thickness and density using clinical routine imaging techniques. Future work will concentrate on investigating how this approach can improve the estimation of mechanical strength of bony structures, the prevention of fracture, and the management of osteoporosis. 


\section{ACKNOWLEDGMENT}

The research leading to these results has received funding from: Programa Estatal de Investigación, Desarrollo e Innovación Orientada a los Retos de la Sociedad, Ministerio de Economía y Competitividad (Reference: RTC-2014-2740-1), Centro para el Desarrollo Tecnológico Industrial, Ministerio de Economía y Competitividad (Eurostars program, Project ID: 9 140) and European Union (Osteoporotic Virtual Physiological Human Project, VPHOP FP7-ICT2008-223865). The work of Ludovic Humbert is supported by: Programa Estatal de Promoción del Talento y su Empleabilidad - Torres Quevedo, Ministerio de Economía y Competitividad (Reference: SPTQ1300X006124XV0). The authors gratefully acknowledge Silvana Di Gregorio and Leen Lenaerts for data collection. 


\section{REFERENCES}

1. O. Johnell and J. A. Kanis, "An estimate of the worldwide prevalence and disability associated with osteoporotic fractures," Osteoporos Int 17, 1726-1733 (2006).

315 2. Y. Bala, R. Zebaze and E. Seeman, "Role of cortical bone in bone fragility," Current opinion in rheumatology 27, 406-413 (2015).

3. V. Bousson, A. Le Bras, F. Roqueplan, Y. Kang, D. Mitton, S. Kolta, C. Bergot, W. Skalli, E. Vicaut, W. Kalender, K. Engelke and J. D. Laredo, "Volumetric quantitative computed tomography of the proximal femur: relationships linking geometric and densitometric variables to bone strength. Role for compact bone," Osteoporos Int 17, 855-864 (2006).

4. D. L. Newman, G. Dougherty, A. al Obaid and H. al Hajrasy, "Limitations of clinical CT in assessing cortical thickness and density," Physics in medicine and biology 43, 619-626 (1998).

5. S. Prevrhal, K. Engelke and W. A. Kalender, "Accuracy limits for the determination of cortical width and density: the influence of object size and CT imaging parameters," Physics in medicine and biology 44, 751-764 (1999).

6. S. Prevrhal, J. C. Fox, J. A. Shepherd and H. K. Genant, "Accuracy of CT-based thickness measurement of thin structures: modeling of limited spatial resolution in all three dimensions," Medical physics 30, 1-8 (2003).

7. E. Rollano-Hijarrubia, R. Stokking, F. van der Meer and W. J. Niessen, "Imaging of small high-density structures in CT A phantom study," Academic radiology 13, 893-908 (2006).

8. A. Pakdel, N. Robert, J. Fialkov, A. Maloul and C. Whyne, "Generalized method for computation of true thickness and x-ray intensity information in highly blurred sub-millimeter bone features in clinical CT images," Physics in medicine and biology $\mathbf{5 7}$, 8099-8116 (2012).

9. G. J. Streekstra, S. D. Strackee, M. Maas, R. ter Wee and H. W. Venema, "Model-based cartilage thickness measurement in the submillimeter range," Medical physics 34, 3562-3570 (2007).

10. G. M. Treece, A. H. Gee, P. M. Mayhew and K. E. Poole, "High resolution cortical bone thickness measurement from clinical CT data," Med Image Anal 14, 276-290 (2010).

11. G. M. Treece, K. E. Poole and A. H. Gee, "Imaging the femoral cortex: thickness, density and mass from clinical CT," Med Image Anal 16, 952-965 (2012).

12. G. M. Treece and A. H. Gee, "Independent measurement of femoral cortical thickness and cortical bone density using clinical CT," Med Image Anal 20, 249-264 (2015).

13. J. Hazrati Marangalou, K. Ito, M. Cataldi, F. Taddei and B. van Rietbergen, "A novel approach to estimate trabecular bone anisotropy using a database approach," Journal of biomechanics 46, 2356-2362 (2013). 
14. J. Moré, "The Levenberg-Marquardt algorithm: Implementation and theory," in Numerical Analysis, Vol. 630, edited by G.

A. Watson (Springer Berlin Heidelberg, 1978), pp. 105-116.

15. P. Besl and N. McKay, "A Method for Registration of 3-D Shapes," IEEE Trans. Pattern Anal. Mach. Intell. 14, 239-256 (1992).

345 16. W. H. O. Scientific Group on Prevention and Management of Osteoporosis, Prevention and management of osteoporosis: report of a WHO Scientific Group. (World Health Organization, Geneva, 2003).

17. J. T. Schousboe, J. A. Shepherd, J. P. Bilezikian and S. Baim, "Executive summary of the 2013 International Society for Clinical Densitometry Position Development Conference on bone densitometry," J Clin Densitom 16, 455-466 (2013).

18. J. Bing and B. C. Vemuri, "Robust Point Set Registration Using Gaussian Mixture Models," Pattern Analysis and Machine Intelligence, IEEE Transactions on 33, 1633-1645 (2011).

19. F. Johannesdottir, T. Turmezei and K. E. Poole, "Cortical bone assessed with clinical computed tomography at the proximal femur," J Bone Miner Res 29, 771-783 (2014).

20. T. Baum, E. Grande Garcia, R. Burgkart, O. Gordijenko, H. Liebl, P. M. Jungmann, M. Gruber, T. Zahel, E. J. Rummeny, S. Waldt and J. S. Bauer, "Osteoporosis imaging: effects of bone preservation on MDCT-based trabecular bone microstructure parameters and finite element models," BMC medical imaging 15, 22 (2015).

21. E. H. van Haaren, B. C. van der Zwaard, A. J. van der Veen, I. C. Heyligers, P. I. Wuisman and T. H. Smit, "Effect of longterm preservation on the mechanical properties of cortical bone in goats," Acta orthopaedica 79, 708-716 (2008).

22. D. M. Black, M. L. Bouxsein, L. M. Marshall, S. R. Cummings, T. F. Lang, J. A. Cauley, K. E. Ensrud, C. M. Nielson and E. S. Orwoll, "Proximal femoral structure and the prediction of hip fracture in men: a large prospective study using QCT," J Bone Miner Res 23, 1326-1333 (2008).

23. V. D. Bousson, J. Adams, K. Engelke, M. Aout, M. Cohen-Solal, C. Bergot, D. Haguenauer, D. Goldberg, K. Champion, R. Aksouh, E. Vicaut and J. D. Laredo, "In vivo discrimination of hip fracture with quantitative computed tomography: Results from the prospective European Femur Fracture Study (EFFECT)," J Bone Miner Res 26, 881-893 (2011).

24. X. Cheng, J. Li, Y. Lu, J. Keyak and T. Lang, "Proximal femoral density and geometry measurements by quantitative computed tomography: association with hip fracture," Bone 40, 169-174 (2007).

25. F. Johannesdottir, K. E. Poole, J. Reeve, K. Siggeirsdottir, T. Aspelund, B. Mogensen, B. Y. Jonsson, S. Sigurdsson, T. B. Harris, V. G. Gudnason and G. Sigurdsson, "Distribution of cortical bone in the femoral neck and hip fracture: a prospective case-control analysis of 143 incident hip fractures; the AGES-REYKJAVIK Study," Bone 48, 1268-1276 (2011). 
26. L. Yang, W. J. Udall, E. V. McCloskey and R. Eastell, "Distribution of bone density and cortical thickness in the proximal 370 femur and their association with hip fracture in postmenopausal women: a quantitative computed tomography study," Osteoporos Int 25, 251-263 (2014). 